\title{
Sepsis and septic shock: can we win the battle against this hidden crisis?
}

\author{
V.G. Dassanayake \\ Department of Surgery, Faculty of Medicine, University of Colombo, Sri Lanka
}

Key words : Sepsis; blood cultures; measure

\section{Introduction}

Sepsis is a global burden. Septic shock is a serious condition and a common reason for admission to the intensive care unit. A prospective cohort study done in patients admitted to intensive care units in 16 Asian countries found that mortality from sepsis induced organ dysfunction was found to be $44.5 \%$. This is an indicator of the magnitude of the problem. Despite advances in technology sepsis remains a costly and often a fatal condition with increased mortality.

The initial definition of sepsis was introduced in 1991 at a consensus conference of experts and was later revisited in 2001. Early this year a third international consensus revised the definition of sepsis and septic shock which has been coined sepsis-3". The basis of the new definition is a greater understanding of the pathophysiology of microcirculatory dysfunction associated with sepsis and septic shock, with the aim of assisting clinicians to recognize this life threatening syndrome early.

\section{Re-defining Sepsis and Septic Shock (Sepsis-3)}

Sepsis (2001) was defined as suspected or documented evidence of infection in the presence of disturbances in any of the following five variables, namely general status, haemodynamic, inflammatory, organ perfusion and tissue perfusion. This was subsequently replaced by its new definition in February 2016. However, these variables may still be useful to the clinician to suspect a patient with sepsis, as it indicates the possibility of ongoing inflammation and organ dysfunction.

Sepsis was re-defined in 2016 as a life threatening organ dysfunction due to a dysregulated host response to infection. Organ dysfunction is defined as an increase (an acute change of 2 points or greater) in the sequential organ failure assessment (SOFA) score (Table 1) [1].

Correspondence: V.G. Dassanayake

E-mail: viharadassanayake@gmail.com

DOI: http://10.4038/sljs.v34i4.8318
Septic shock is a subset of sepsis with a high mortality. It has been defined as fluid unresponsiveness, hypotension, elevated serum lactate levels $>2 \mathrm{mmol} / \mathrm{L}(>18 \mathrm{mg} / \mathrm{dl})$ and the need for vasopressors to maintain a mean arterial pressure of $65 \mathrm{mmHg}$ or greater [1].

Absent from the 2016 definition is the term "severe sepsis", as mortality rates from sepsis alone can be $10 \%$ or more making this condition already severe [1].

\section{q-SOFA: let us not disregard this index}

The consensus document has also introduced a bedside index termed as quick SOFA (Table 2) comprising of three variables to enable clinicians to identify patients with suspected infection who are been treated outside critical care units and likely to develop complications of sepsis [1].

In the presence of more than 2 variables, the patient is considered to be at risk of sepsis. q-SOFA can be useful in a resource limited setting as it does not require advanced monitoring and it is not labour intensive. This index can be used to "track and trigger", similar to the early warning score. It is important to note that q-SOFA per se is not a test for sepsis and is subjective, which can be a limiting factor in defining patients with sepsis.

\section{Management bundles: What do the guidelines say?}

The Surviving Sepsis Campaign (SSC) guideline is an international effort to promote awareness, improve patient care and outcome following sepsis [2].

Early recognition and timely intervention together with the implementation of the " 3 hour resuscitation bundle" and the "6 hour septic shock management bundle" can improve patient outcome [2]. A bundle is a selected set of elements of care for a group of interventions distilled from evidencebased practice, and when implemented together, within a specified time frame, have an effect on outcome beyond that of an individual element/s.

It is important to remind ourselves that this is only a guide, and even though it has become a standard of care, some aspects of patient care in these guidelines have been 
questioned by many, and is under scrutiny.

Clinicians must bear in mind that the haemodynamic profile of each patient presenting with sepsis/ septic shock may be different and therapeutic intervention/s need to be tailored and modified based on these changes [3].

\section{3 hour resuscitation bundle}

The following steps must be completed within the first 3 hours from the time of presentation of a patient to the emergency department with suspected sepsis [4].

1. Measure lactate levels

2. Obtain blood cultures prior to administration of antibiotics

3. Administer broad spectrum antibiotics

4. Administer $30 \mathrm{ml} / \mathrm{kg}$ of crystalloids for hypotension or lactate $>4 \mathrm{mmol} / \mathrm{L}$

\section{Lactate in sepsis}

An elevated level of lactate in patients with sepsis indicates disease severity. It has been postulated that elevated lactate levels are mostly due to activation of the stress response and release of norepinephrine and is not merely due to anaerobic metabolism [5]. Failure of blood lactate levels to decline in response to treatment indicates a poorer outcome.

\section{Blood cultures (other sites as appropriate) and antibiotics: hit them early and hit them hard}

Cultures must be obtained prior to commencing antibiotic therapy, provided that there is no significant delay $(>45$ minutes) in the start of antimicrobials (grade 1C) [2]. It is imperative to obtain blood cultures from two different sites within minutes of each other and to aerobic and anaerobic blood culture bottles. If an invasive device has been in situ for $>48$ hours at least one sample must be obtained through the line in situ and other concurrently from a peripheral site. ${ }^{2}$ Early and appropriate antibiotic therapy must be instituted within the first hour of recognition of septic shock (grade 1 B). Appropriate antibiotic should target the most likely pathogen based on the site of infection. It has been found that for each hours delay in administering antibiotics in septic shock, mortality increases by $7.6 \%$ [6].

Response to treatment must be re-assessed after 48 - 72 hours and de-escalate therapy as appropriate. A decline in serum biomarkers such as procalcitonin may help clinicians in decision making regarding de-escalation of antimicrobial treatment.

\begin{tabular}{|c|c|c|c|c|c|}
\hline \multirow[b]{2}{*}{ System } & \multicolumn{5}{|l|}{ Score } \\
\hline & 0 & 1 & 2 & 3 & 4 \\
\hline \multicolumn{6}{|l|}{ Respiration } \\
\hline $\begin{array}{l}\mathrm{PaO}_{2} / \mathrm{FlO}_{2}, \mathrm{~mm} \mathrm{Hg} \\
(\mathrm{kPa})\end{array}$ & $2400(53.3)$ & $<400(53.3)$ & $<300(40)$ & $\begin{array}{l}<200(26.7) \text { with } \\
\text { respiratory support }\end{array}$ & $\begin{array}{l}<100(13.3) \text { with } \\
\text { respiratory support }\end{array}$ \\
\hline \multicolumn{6}{|l|}{ Coagulation } \\
\hline Platelets, $\times 10^{3} / \mu \mathrm{L}$ & $\geq 150$ & $<150$ & $<100$ & $<50$ & $<20$ \\
\hline \multicolumn{6}{|l|}{ Liver } \\
\hline $\begin{array}{l}\text { Bilinubin, mg/dl } \\
\text { (umol/L) }\end{array}$ & $<1.2(20)$ & $1.2 \cdot 1.9(20 \cdot 32)$ & $2.0-5.9(33-101)$ & $6.0-11.9(102-204)$ & $>12.0(204)$ \\
\hline Cardiovascular & MAP $\geq 70 \mathrm{~mm} \mathrm{Hg}$ & MAP $<70 \mathrm{~mm} \mathrm{Hg}$ & $\begin{array}{l}\text { Dopamine }<5 \text { or } \\
\text { dobutamine (any dose) }\end{array}$ & $\begin{array}{l}\text { Dopamine } 5.1-15 \\
\text { or epinephrine } \leq 0.1 \\
\text { or norepinephrine } \leq 0.1^{\circ}\end{array}$ & $\begin{array}{l}\text { Dopamine }>15 \text { or } \\
\text { epinephrine }>0.1 \\
\text { or norepinephrine }>0.1^{\circ}\end{array}$ \\
\hline \multicolumn{6}{|l|}{ Central nervous system } \\
\hline $\begin{array}{l}\text { Glasgow Coma Scale } \\
\text { score }\end{array}$ & 15 & $13-14$ & $10-12$ & $6-9$ & $<6$ \\
\hline \multicolumn{6}{|l|}{ Renal } \\
\hline $\begin{array}{l}\text { Creatinine, mg/dl } \\
\text { ( } \mu \mathrm{mol} / \mathrm{L})\end{array}$ & $<1.2(110)$ & $1.2-1.9(110-170)$ & $2.0-3.4(171-299)$ & $3.5-4.9(300-440)$ & $>5.0(440)$ \\
\hline Urine output, $\mathrm{mL} / \mathrm{d}$ & & & & $<500$ & $<200$ \\
\hline \multirow{2}{*}{\multicolumn{3}{|c|}{$\begin{array}{l}\text { Abbreviations: } \mathrm{FlO}_{2} \text {, fraction of inspired oxygen; MAP, mean arterial pressure: } \\
\mathrm{PaO}_{2} \text {, partial pressure of oxygen. }\end{array}$}} & \multicolumn{3}{|c|}{${ }^{\mathrm{b}} \mathrm{Catecholamine} \mathrm{doses} \mathrm{are} \mathrm{given} \mathrm{as} \mu \mathrm{g} / \mathrm{kg} / \mathrm{min}$ for at least 1 hour. } \\
\hline & & & \multicolumn{3}{|c|}{$\begin{array}{l}\text { 'Glasgow Coma Scale scores range from 3-15; higher score indicates better } \\
\text { neurological function. }\end{array}$} \\
\hline
\end{tabular}

Table 1 Sequential Organ Failure Assessment Score (SOFA): Reproduced from http://www.jamasepsis.com 


\begin{tabular}{|c|c|}
\hline Variable & Finding \\
\hline mental status & altered \\
\hline systolic blood pressure & $<100 \mathrm{mmHg}$ \\
\hline respiratory rate & $>22$ breaths per minute \\
\hline
\end{tabular}

Table 2 q-SOFA Score

\section{Fluid resuscitation: a challenging task}

The guideline recommends an initial fluid bolus of crystalloids such as $0.9 \%$ normal saline $30 \mathrm{ml} / \mathrm{kg}$ as the initial fluid of choice in the resuscitation of severe sepsis and septic shock (grade 1B). Further incremental boluses can be considered until there is improvement in haemodynamic parameters (grade 1C) [2]. If excessive volumes of crystalloids are needed albumin can be considered during resuscitation. The exact volume and concentration of albumin has not been clearly elucidated.

Hydroxyethyl starch should not be used as resuscitation fluid when managing septic patients as acute kidney injury requiring renal replacement therapy has been observed in patients receiving high molecular weight starch solutions [2].

Hypo-perfusion in septic shock is multifactorial. Venous and arterial dilatation, intravascular volume depletion and sepsis induced myocardial dysfunction with ventricular dilatation and a reduced ejection fraction can cause hypo-perfusion to vital organs. This clearly indicates that vigilant monitoring is required during fluid resuscitation and it is important to appreciate that fluids alone will not help achieve the desired targets [7].

The release of inflammatory mediators in sepsis, results in increased capillary leakage and damage to the endothelial glycocalyx thereby predisposing to fluid overload. Insufficient fluid replacement will result in inadequate tissue perfusion [7].

Therefore administering the right amount of the right type of fluid to the right patient is of paramount importance. Haemodynamic profile of each patient should be dynamically monitored [3]. The clinician needs to be cautious when administering fluids to the elderly with limited cardiac reserves and to patients with sepsis induced myocarditis. Therefore the bolus dose of $30 \mathrm{ml} / \mathrm{kg}$ of crystalloid does not "fit them all".

\section{6 hour septic shock management bundle}

According to the surviving sepsis campaign guideline 6 hour bundle (updated in 2015), the following steps must be undertaken within the stipulated time frame.

Vasopressors must be commenced for hypotension that is unresponsive to fluids to target a mean arterial pressure (MAP) of $65 \mathrm{mmHg}$ or greater and in the event of persistent hypotension despite fluids with an initial serum lactate level of more than $4 \mathrm{mmol} / \mathrm{L}$. In such situations the volume status must be re-assessed and lactate levels must be re-measured [2].

Assessment of volume status and tissue perfusion can be accomplished by repeated focused examination of vital signs or by employing any two of the following measures within 6 hours of presentation [2].

- Measure central venous pressure (CVP)

- Measure central venous oxygen saturation $\left(\mathrm{ScVO}_{2}\right)$

- Bedside cardiovascular ultrasound (to assess IVC diameter, left ventricular function and anterior lung examination to detect volume overload)

- Dynamic assessment of fluid responsiveness with passive leg raising or fluid challenge test

The six hour management bundle was revised following the publication of results of three randomized controlled trials ProCESS, ARISE and ProMISE. These trials failed to demonstrate superiority of use of a central venous catheter to monitor CVP and $\mathrm{ScVO}_{2}$ in patients who had received timely antibiotics and appropriate fluid resuscitation compared with controls $[8,9,10]$. Based on these three trials it does not mandate central lines as a part of an early resuscitation strategy.

CVP indicates pressure in the right heart and does not reflect intravascular volume nor does it predict fluid responsiveness. Therefore it is evident that it has minimal or no role in resuscitating a septic patient $[3,11]$.

It is important to appreciate that hypovolaemia may not be the only indication for IVC collapsibility with inspiration on ultrasound imaging, as other factors such as intra-abdominal pressure and variables such as venous return can also contribute to changes in IVC diameter. Therefore fluid responsiveness with the aid of IVC diameter per se may not be the best option.

Functional haemodynamic monitoring would be the ideal to determine the response to a fluid challenge and should ideally be done using a bedside echocardiogram. Not all patients in septic shock will be fluid responsive. Patients with sepsis induced cardiomyopathy might be a high risk group, therefore giving fluids will not be the best option in this vulnerable patient population [12]. Therefore it is logical to use invasive 
cardiac monitoring and tailor the resuscitation strategy to achieve the desired haemodynamic goals.

\section{Vasopressors and inotropes in septic shock}

Norepinephrine is considered to be the vasopressor of choice in septic shock. Decision to commence norepinephrine should be made early as norepinephrine constricts both arteries and veins thereby increasing the preload and counteracting the vasodilated state induced by sepsis.

Evidence suggests that early restoration of blood pressure within the auto - regulatory values with the use of norepinephrine is mandated in the presence of sepsis induced acute kidney injury (AKI) [12]. Addition of vasopressin $(0.03 \mathrm{U} /$ minute $)$ is advocated in the existing guidelines with the intent of decreasing the norepinephrine dose or increasing the MAP, but it should never be administered as the sole agent.

Epinephrine can be added or substituted for norepinephrine when needed [2].

Use of dopamine to counteract the vasodilation induced by sepsis and low dose regimes in AKI cannot be recommended [12]. Dopamine may be used in patient with low heart rates and who are at reduced risk of developing arrhythmias (grade 2C) [2]. Dobutamine can be added to the existing regime in the presence of myocardial dysfunction or in the presence of on-going tissue hypo-perfusion.

\section{Management beyond the initial moments}

It is evident that patients with septic shock will require intensive care treatment as monitoring vital signs and parameters become vital.

Source identification and eradication is imperative. Use of low dose steroids (200mg of hydrocortisone over 24 hours) have been recommended in the current guidelines when septic shock is refractory to treatment and the inotrope requirements are high [2]. However there is no convincing data to state that steroids improve mortality [13].

Acceptable haemoglobin levels in adult septic patients in the absence of ischaemic coronary artery disease, acute haemorrhage and severe hypoxaemia is $7-9 \mathrm{~g} / \mathrm{dl}$. Platelets should be transfused when the counts are $10,000 / \mathrm{mm}^{3}$ in the absence of apparent bleeding and a count of $50,000 / \mathrm{mm}^{3}$ is required for surgical and invasive procedures. Fresh frozen plasma should not be transfused to treat laboratory reports in the absence of obvious bleeding.

ICU care bundles such as prophylaxis for deep vein thrombosis either by pharmacological or non-pharmacological methods, stress ulcer prophylaxis for the high risk patient, preferably with proton pump inhibitors should be commenced. Sepsis induced ARDS requires lung protective ventilation with low tidal volume ventilation. Blood glucose values should be kept below $180 \mathrm{mg} / \mathrm{dl}$ but tight glycaemic control is not advocated. Early initiation of feeding is recommended in the critically ill septic patient.

\section{Conclusion}

Diagnosis of sepsis and septic shock is primarily clinical. Prompt diagnosis entails a detailed history and physical examination to identify the potential source of infection.

Restoring adequate circulation, early antibiotic therapy, source identification and supportive care are essential for a successful outcome.

Clear guidance for recognition of early warning signs of sepsis, interventions and escalation must be in place in every medical institution as early recognition and timely interventions can save lives.

\section{Acknowledgements}

I am grateful to Professor Anuja Abayadeera for critically analysing the intellectual content and for the advice given.

All authors disclose no conflict of interest. The study was conducted in accordance with the ethical standards of the relevant institutional or national ethics committee and the Helsinki Declaration of 1975, as revised in 2000 .

\section{References}

1. The $3^{\text {rd }}$ International Consensus definition of sepsis and septic shock. JAMA 2016;315(8): 757-759

2. Surviving Sepsis Campaign guidelines for the management of severe sepsis and septic shock: 2012. Crit Care Med 2013; 41(2): 580-637 https://doi.org/10.1097/CCM.0b013e31827e83af

3. Marik PE, Monnet X, Teboul JL. Haemodynamic parameters to guide fluid therapy. Annals of Intensive Care 2011;1(1) https://doi.org/10.1186/2110-5820-1-1

4. Surviving Sepsis Campaign Bundles. Available from: https://www.survivingsepsis.org/bundles/pages/default.aspx (Accessed date 28/09/2016)

5. Marik PE, Bellomo R. Lactate clearance as a target therapy in sepsis: a flawed paradigm. Crit Care Med 2013; 1(1):3 https://doi.org/10.13172/2052-9309-1-1-431

6. Ngyuen H B, Rivers E P, Knoblich B P et al. Early lactate clearance is associated with impaired with outcome in sepsis and septic shock. Crit Care Med 2004; 32: 1637-1642 https://doi.org/10.1097/01.CCM.0000132904.35713.A7

7. Accelerated goal directed therapy for septic shock. Available from: https://www.emcrit.org/pilmcrit/accelerated-goaldirected-therapy for septic shock (Accessed date 16/09/2016)

8. ProCESS Investigators, Yealy DM, Kellum JA, Juang DT et al. A randomized trial of protocol-based care for early septic shock. N Engl J med 2014;370(18) 1683-169310. Richardson LA, Jones GW. A review of the reliability and validity of the Edmonton Symptom Assessment System. Curr Oncol 2009; 16(1): 55 . 
https://doi.org/10.1056/NEJMoa1401602

9. The ARISE Investigators and the ANZICS Clinical Trials Group. Goal directed resuscitation for patients with early septic shock. NEngl J Med;371:1496-1506

https://doi.org/10.1056/NEJMoa1404380

10. Mouncey PR, Osborn TM, Power GS et al for the ProMISE trial investigators. Trial of early goal directed resuscitation for septic shock. N Engl J Med 2015:

doi;10.1056/NEJMoa1500896

11. Marik PE, Baram M, Valid B. Does the CVP predict fluid responsiveness? A systematic review of the literature and the tale of seven mares. Chest 2008; 134:172-178

https://doi.org/10.1378/chest.07-2331

12.Bellomo R, Wan L, May C. Vasoactive drugs and AKI. Crit Care Med 2008; 34(4): 179-86 https://doi.org/10.1097/CCM.0b013e318169167f

13. Steroids in septic shock : four misconceptions and one truth. Available from: https://www.emcrit.org/pulmcrit/steroids-inseptic-shock-four misconceptions-and-one-truth (Accessed date 17/09/2016) 